\title{
On Existence of Solitons for the Second Harmonic Equations of a Laser Beam
}

\author{
V. CaO Long ${ }^{a}$, P.P. Goldstein ${ }^{b, *}$ And S. Vu NGOC ${ }^{c}$ \\ ${ }^{a}$ Institute of Physics, University of Zielona Góra \\ Podgórna 50, 65-246 Zielona Góra, Poland \\ ${ }^{b}$ The Andrzej Soltan Institute for Nuclear Studies \\ Hoża 69, 00-681 Warsaw, Poland \\ ${ }^{c}$ Faculty of High Technology, Vinh University, Nghe An, Vietnam
}

(Received August 17, 2004)

\begin{abstract}
We look for conditions of existence of soliton solutions for equations governing propagation of a monochromatic laser beam coupled to its second harmonic in a nonlinear medium. The system proves to be non-integrable in the sense of Painlevé. However it is partially integrable for some values of its parameters. We further check the possibility of solving the equations by the Hirota bilinear method. The system is found to be solvable this way provided that amplitudes of both modes are equal while the complex phase of the second harmonic is equal to the double phase of the fundamental mode (modulo $\pi$ ). The Hirota scheme is found to work merely for exact resonance, i.e. for the ratio of the dispersion coefficients equal to the ratio of frequencies. Finally, all these conditions may only be satisfied by single envelope travelling waves, in which the envelope has locally the shape of the Weierstrass $\wp$ function.
\end{abstract}

PACS numbers: 42.81.Dp

\section{Introduction}

Since the pioneering paper [1] where the experimental observation of frequency doubling, or otherwise second harmonic generation was presented, this first phenomenon of nonlinear optics has steadily been a subject of active research. It is well known that a great deal of the physical processes involved in a

*corresponding author; e-mail: Piotr.Goldstein@fuw.edu.pl 
given nonlinear problem can be understood in terms of the formation of spatial, temporal or spatio-temporal localized structures or solitons. The study of these localized waves is strongly complicated by the fact that the nonlinear equations of the given system are not integrable as in our case of second harmonic generation. With the exception of one analytical solution [2] the solitary wave solutions have to be determined numerically [3].

In a previous paper [4] we looked for conditions enabling the existence of solitons when the fundamental mode of a laser beam interacted with its third harmonic. We found that soliton solutions of the Hirota type existed if and only if the complex phase of the third harmonic was equal to the tripled phase of the fundamental mode (modulo $\pi$ ) while the amplitudes of these modes remained in a special proportion. This was possible only for exact resonance, i.e. for the ratio of the dispersion coefficients equal to the ratio of frequencies; moreover all these conditions could only be satisfied for single envelope solitons of the cubic Schrödinger type. Possibility of such solutions was foreseen in that paper [4] due to the partial Painlevé property [5] of those equations, although the equations were not Painlevé integrable [6].

In this paper we perform a similar calculation for the second harmonic equations. The equations ([7] without the mismatch and damping) are based on a quadratically nonlinear interaction of the wave with a medium. This system describes simultaneously the case of self-guided beams in bulk materials and the case of planar waveguides. The results resemble those for the third harmonic. Firstly, the system is found to be non-integrable in the sense of Painlevé [6]. Secondly, the Hirota bilinear method [8] yields a solvable system only if the resonance is exact, i.e. the ratio of the dispersion coefficients for the fundamental mode and its second harmonic is exactly 2. For such dispersion coefficients there exist solutions in which amplitudes of both modes are equal to each other, while the phase of the second harmonic is equal to the double phase of the fundamental mode. As in the 3rd harmonic case, this system admits only single travelling wave solutions. However the shape of those solutions is entirely different from the cubic Schrödinger solitons: namely the wave envelope is proportional to the Weierstrass $\wp$ function of a rescaled time and thus it is a wave train rather than a solitary wave. Also unlike the third harmonic equations, which have the partial Painlevé property [4], these equations are partially integrable in the sense of Painlevé merely for special ratios of the dispersion coefficients $(2$ and -1 ; there are no travelling wave solutions in the latter case).

The paper begins with derivation of the equations governing propagation of our modes in Sec. 2; Sec. 3 is devoted to their Painlevé analysis while the Hirota method is applied to these equations in Sec. 4. In Sec. 5 we find the travelling wave solution explicitly and discuss its properties. 


\section{The second harmonic equations}

Starting from Maxwell's equations we obtain the wave equation for propagating electric field $\boldsymbol{E}(\boldsymbol{x}, t)$

$$
\left(\nabla_{\perp}^{2}+\frac{\partial^{2}}{\partial z^{2}}-\frac{1}{c^{2}} \frac{\partial^{2}}{\partial t^{2}}\right) \boldsymbol{E}(\boldsymbol{x}, t)=\frac{1}{\varepsilon_{0} c^{2}} \frac{\partial^{2}}{\partial t^{2}}\left(\boldsymbol{P}^{\mathrm{L}}(\boldsymbol{x}, t)+\boldsymbol{P}^{\mathrm{NL}}(\boldsymbol{x}, t)\right)
$$

where $z$ is the direction of propagation, $\nabla_{\perp}^{2}$ is the transverse part of the Laplacian, while $\boldsymbol{P}^{\mathrm{L}}$ and $\boldsymbol{P}^{\mathrm{NL}}$ are linear and nonlinear polarization vectors, respectively.

We are interested in the resonant interaction between a linearly polarized beam of frequency $\omega_{0}$ and its second harmonic, which is assumed to be identically polarized. For the case of a dielectric medium with nonlinear susceptibility $\chi^{(2)}$ we have in the frequency space

$$
\begin{aligned}
& \tilde{P}_{i}^{\mathrm{NL}}(\boldsymbol{x}, \omega)=\frac{\varepsilon_{0}}{2 \pi} \iint \mathrm{d} \omega_{1} \mathrm{~d} \omega_{2} \chi_{i j k}^{(2)}\left(-\left(\omega_{1}+\omega_{2}\right) ; \omega_{1}, \omega_{2}\right) \tilde{E}_{j}\left(\boldsymbol{x}, \omega_{1}\right) \\
& \times \tilde{E}_{k}\left(\boldsymbol{x}, \omega_{2}\right) \delta\left(\omega_{1}+\omega_{2}-\omega\right),
\end{aligned}
$$

where the tilde denotes quantities in the Fourier space.

Now we assume the electric field to have the form of a sum of two modes whose frequencies are $\omega_{0}$ (the fundamental mode) and $2 \omega_{0}$ (the second harmonic). If the amplitudes of these modes vary slowly in comparison with $\omega_{0}$, we can write the evolution equations in a reference frame moving with the group velocity of the fundamental beam [7] as

$$
\begin{aligned}
& \mathrm{i} \frac{\partial A_{1}}{\partial z}+\frac{D_{1}}{2} \frac{\partial^{2} A_{1}}{\partial t^{2}}+\chi_{1} A_{1}^{*} A_{2}=0, \\
& \mathrm{i} \frac{\partial A_{2}}{\partial z}+\frac{D_{2}}{2} \frac{\partial^{2} A_{2}}{\partial t^{2}}+\chi_{2} A_{1}^{2}=0 .
\end{aligned}
$$

Here $z$ denotes the position in the reference frame of the wave, the functions of $z$ and $t$ denoted by $A_{1}$ and $A_{2}$ are slowly varying amplitudes of the fundamental frequency and its second harmonic, respectively, the constants $D_{1}$ and $D_{2}$ characterize their dispersion, while the other constant parameters $\chi_{1}$ and $\chi_{2}$ measure the size of nonlinear coupling between them, $\chi_{1} \equiv\left(\omega_{0}^{2} / \varepsilon_{0} k_{1} c^{2}\right) \chi^{(2)}\left(-\omega_{0} ; 2 \omega_{0},-\omega_{0}\right)$ and $\chi_{2} \equiv\left(\omega_{0}^{2} / \varepsilon_{0} k_{2} c^{2}\right) \chi^{(2)}\left(-2 \omega_{0} ; \omega_{0}, \omega_{0}\right)$, where $\chi^{(2)}\left(\omega_{1}+\omega_{2} ;-\omega_{1},-\omega_{2}\right)$ is the coefficient of the appropriate nonlinear coupling, $k_{1}$ and $k_{2}$ are the wave vectors of the harmonic. If we denote the dispersion relations of the fundamental and second harmonics respectively by $\beta_{1}(\omega)=0$ and $\beta_{2}(\omega)=0$, then the dispersion coefficients $D_{n}, n=1,2$ are expressed by [7]

$$
D_{n}=-\left.\frac{\partial^{2} \beta_{n}}{\partial \omega^{2}}\right|_{\omega=\omega_{0}}
$$

In (3) we neglected the phase mismatch and we assumed that the group velocities of the two pulses (fundamental and second harmonics) were equal, so the tem- 
poral walk-off term disappeared. The spatial walk-off can be avoided in a planar waveguide. The stationary beam propagation in planar waveguides is considered as the phenomenon similar to the pulse propagation in fibers. This is so-called spatio-temporal analogy [9]. Thus our results will also be adequate to the case of a planar waveguide. Positive and negative $D_{n}$ correspond to anomalous and normal dispersion, respectively. It is worthwhile to reduce the number of arbitrary parameters by rescaling the time: $\sqrt{2 / D_{1}} t_{\text {old }}=t_{\text {new }}$ and reverting $z$ if necessary: $z_{\text {old }}=\operatorname{sign}\left(D_{1}\right) z_{\text {new }}$. If we denote

$$
\sqrt{\left|\chi_{1} \chi_{2}\right|} A_{1}=U, \quad \operatorname{sign}\left(D_{1}\right) \chi_{1} A_{2}=W,
$$

then the system (4) may be written in a simple form

$$
\begin{aligned}
& \mathrm{i} U_{, z}+U_{, t t}+U^{*} W=0, \\
& \mathrm{i} W_{, z}+P W_{, t t} \pm U^{2}=0,
\end{aligned}
$$

where $P=D_{2} / D_{1}$ while the sign \pm in front of $U^{2}$ in the second equation is just the sign $\left(\chi_{1} \chi_{2}\right)$, which corresponds to positive and negative coupling between the modes, respectively. Subscripts following a comma denote differentiation.

Note that we have admitted reversal of the sign of $z$ when the fundamental mode has negative dispersion. This transformation of the physical picture to its mirror image does not influence integrability and existence of solitons.

\section{The Painlevé test}

We perform the Painlevé test (which should rather be named after Kovalevskaya [10]) in its version extended for partial differential equations $[11,5]$. A comprehensive review of its interpretations, methods, and validity was given in [6] for the ordinary differential equations and [12] for the partial differential equations. A pedestrian's approach may be found in [13].

The standard procedure begins with analytical extension of the examined equations to the complex $(z, t)$ biplane. Since the complex conjugates of $U, W$ have analytic extensions of their own, we treat them as extra unknowns, $V=U^{*}$ and $Y=W^{*}$, respectively. This way our equations (6) become a $4 \times 4$ system

$$
\begin{aligned}
& \mathrm{i} U,_{z}+U,_{t t}+V W=0, \\
& -\mathrm{i} V,_{z}+V,_{t t}+U Y=0, \\
& \mathrm{i} W,_{z}+P W,_{t t} \pm U^{2}=0, \\
& -\mathrm{i} Y,_{z}+P Y,_{t t} \pm V^{2}=0 .
\end{aligned}
$$

For the purpose of the Painlevé test it is sufficient to consider the case with the plus sign in front of $U^{2}$ and $V^{2}$. The case with the minus sign may be obtained by a change of dependent variables $U_{\text {new }}=\mathrm{i} U_{\text {old }}, V_{\text {new }}=\mathrm{i} V_{\text {old }}$. 
In the method of [11] we assume the general solution of a system with $n$ independent variables in the form of a Laurent series in a neighborhood of an $n-1$ dimensional singular manifold (rather than a singular point), calculate the initial terms and determine the subsequent terms from recurrence formulae. The recurrence formulae are simplest when we choose the expansion variable $F$ in the Kruskal gauge [14], i.e. we assume that the equation of the manifold $F(x, t)=0$ has the form already solved with respect to one of the variables, e.g. $t$. Thus for the sake of simplicity we perform the Laurent expansion in

$$
F(x, t)=t-\zeta(z)
$$

where $\zeta$ is an arbitrary function of $z$, and assume that the expansion coefficients depend on $z$ only.

Let the four Laurent series be

$$
\begin{aligned}
& U=F^{p} \sum_{n=0}^{\infty} U_{n} F^{n}, \quad V=F^{q} \sum_{n=0}^{\infty} V_{n} F^{n}, \\
& W=F^{r} \sum_{n=0}^{\infty} W_{n} F^{n}, \quad Y=F^{s} \sum_{n=0}^{\infty} Y_{n} F^{n} .
\end{aligned}
$$

We substitute them to (7) and look for solutions of the recurrence relations resulting from comparison of the coefficients at subsequent powers of $F$.

The leading order exponents are $p=q=r=s=-2$. Two non-Painlevé families for which

$$
p=-2 \pm \sqrt{3} \mathrm{i}, \quad q=-2 \mp \sqrt{3} \mathrm{i}, \quad r=-2 \pm 2 \sqrt{3} \mathrm{i}, \quad s=-2 \mp 2 \sqrt{3} \mathrm{i}
$$

also exist. We concentrate on the previous family as it is the only one promising meromorphic soliton solutions. The coefficients at $F^{-2}$ read respectively

$$
U_{0}= \pm 6 \sqrt{P} / Q_{0}, \quad V_{0}= \pm 6 \sqrt{P} Q_{0}, \quad W_{0}=-6 / Q_{0}^{2}, \quad Y_{0}=-6 Q_{0}^{2},
$$

where $Q_{0}$ is an arbitrary function of $z$. The indices $n$ ("resonances"), at which other arbitrary functions may be obtained, follow from the requirement that the determinant of the linear system

$$
\begin{aligned}
& \left(6-5 n+n^{2}\right) U_{n}+W_{0} V_{n}+V_{0} W_{n}=0, \\
& Y_{0} U_{n}+\left(6-5 n+n^{2}\right) V_{n}+U_{0} Y_{n}=0, \\
& 2 U_{0} U_{n}+\left(6-5 n+n^{2}\right) P W_{n}=0, \\
& 2 V_{0} V_{n}+\left(6-5 n+n^{2}\right) P Y_{n}=0
\end{aligned}
$$

vanish. This condition yields the indicial equation, which proves to be an algebraic equation of 8 th degree for $n$. Its roots are

$$
-1 ; 0 ; 5 ; 6 ;(5-\mathrm{i} \sqrt{23}) / 2 ;(5+\mathrm{i} \sqrt{23}) / 2 ;(5-\mathrm{i} \sqrt{47}) / 2 ;(5+\mathrm{i} \sqrt{47}) / 2 .
$$


Except for the root -1 which corresponds to the arbitrariness of $F$, all other roots should be nonnegative integers, if the system is to be Painlevé integrable. Otherwise the number of arbitrary coefficients (i.e. first integrals) is smaller than the order of the equations, and the Laurent series cannot be the general solution (some exceptions with negative indices are treated in [15], [16], and [6]). The occurrence of four complex roots in (13), as well as the existence of a non-Painlevé family of the leading order coefficients (10) mean that our system is non-Painlevé. Still it may have a four-parameter class of meromorphic solutions, which could encompass one- or even two-soliton solutions, provided that the compatibility conditions at the indices $n=5$ and $n=6$ are satisfied. Such a situation is known as "partial integrability" [5]. In order to check these compatibility conditions we first have to calculate the coefficients at $n=1$ to 4 . We here skip those details of the calculations. At $n=5$ we get a system of equations which are linearly dependent provided that

$$
P=1 / 2 \text { or } P=-1 \text {. }
$$

If neither of the conditions (14) is satisfied the linear dependence affects the left hand sides only and the system at $n=5$ is contradictory. The compatibility condition at $n=6$ is satisfied for both above values of $P$, which means that we may expect the existence of soliton solutions when

$$
D_{1}=2 D_{2} \quad \text { or } \quad D_{1}=-D_{2} .
$$

This is a constraint on the dispersion coefficients. Solitons are likely to occur when the dispersion of the second harmonic is half of that of the fundamental mode or if the dispersion is exactly opposite for these modes.

\section{The Hirota bilinear method}

The Hirota method [8] relies on a transformation of the original equation, by an appropriate substitution, to a bilinear form

$$
Q\left(D_{z}, D_{t}\right) F \cdot G=0,
$$

where $Q$ is a polynomial in the differential operators $D_{z}, D_{t}$, defined by

$$
D_{z}^{m} D_{t}^{n} F \cdot G=\left.\left(\frac{\partial}{\partial z}-\frac{\partial}{\partial z^{\prime}}\right)^{m}\left(\frac{\partial}{\partial t}-\frac{\partial}{\partial t^{\prime}}\right)^{n} F(z, t) G\left(z^{\prime}, t^{\prime}\right)\right|_{z^{\prime}=z, t^{\prime}=t}
$$

In our case the proper transformation of (6) is

$$
U=G / F, \quad W=H / F
$$

where $G$ and $H$ may be complex while $F$ is assumed to be real. The solution is later sought in a perturbative scheme

$$
F=1+\epsilon^{2} f^{(2)}+\ldots, G=\epsilon g^{(1)}+\epsilon^{3} g^{(3)}+\ldots, H=\epsilon h^{(1)}+\epsilon^{3} h^{(3)}+\ldots,
$$


where $f, g, h$ are assumed in the form of a sum of exponential or a polynomial functions in both $z$ and $t$. Substitution (18) transforms our equations into

$$
\begin{aligned}
& F\left(\mathrm{i} D_{z}+D_{t}^{2}\right) G \cdot F-G\left(D_{t}^{2} F \cdot F-F G^{*} H / G\right)=0, \\
& F\left(\mathrm{i} D_{z}+P D_{t}^{2}\right) H \cdot F-H\left(P D_{t}^{2} F \cdot F \mp F G^{2} / H\right)=0,
\end{aligned}
$$

where the minus sign in front of $F G^{2} / H$ corresponds to the plus sign in (6) and vice versa.

To get the Hirota bilinear equations we have to assume that all the expressions in the parentheses are equal to zero. However, the system of equations obtained this way is overdetermined. Among others, $D_{t}^{2} F \cdot F$ should at the same time be equal to $F G^{*} H / G$ and $F G^{2} /(P H)$, whence

$$
F G^{*} H / G=F G^{2} /(P H) \in \mathcal{R}
$$

(both hand sides of (21) should be real as $D_{t}^{2} F \cdot F$ is real due to the reality of $F$ ). By writing this relation in the polar coordinates

$$
G=|G| \exp (\mathrm{i} \varepsilon), \quad H=|H| \exp (\mathrm{i} \psi),
$$

we arrive to the conclusion that

$$
|G|=|H| \sqrt{|P|}
$$

and, depending on the sign in (20) and on the sign of $P$

$$
\psi=2 \varphi+k \pi \quad \text { or } \quad \psi=2 \varphi+(k+1 / 2) \pi .
$$

Here $k$ may be an arbitrary integer. The first choice corresponds to the upper sign in (20) when $P>0$ and the lower one when $P<0$, i.e. to the case $D_{1} D_{2} \chi_{1} \chi_{2}>0$, whereas the second choice to the opposite signs of either dispersion or nonlinear coupling of the fundamental mode and its second harmonic. However, upon the second choice the expressions in (21) are purely imaginary rather than the required real quantities. Hence only the first option makes sense.

When the first parentheses in both equations of (20) are combined with their respective complex conjugates, we obtain two conservation laws for the photon number of the modes $G G^{*}$ and $H H^{*}$, respectively

$$
\begin{aligned}
& \frac{\partial}{\partial z} \frac{G G^{*}}{F^{2}}+2 \frac{\partial}{\partial t}\left(\frac{G G^{*}}{F^{2}} \frac{\partial \varphi}{\partial t}\right)=0, \\
& \frac{\partial}{\partial z} \frac{H H^{*}}{F^{2}}+2 P \frac{\partial}{\partial t}\left(\frac{H H^{*}}{F^{2}} \frac{\partial \psi}{\partial t}\right)=0 .
\end{aligned}
$$

Substitution of the conditions (23) and (24) to (25) yields among others

$$
(2 P-1) \frac{\partial}{\partial t}\left(\frac{G G^{*}}{F^{2}} \frac{\partial \varphi}{\partial t}\right)=0
$$

which has nontrivial solutions if and only if 


$$
P=1 / 2,
$$

whence only the upper sign in (20) and (6) is physically sensible.

The result (27) is in accordance with one of the conditions of partial Painlevé integrability (14). On the other hand, an attempt to solve the real parts of the equations (20) for the case when all these conditions $(23,27)$ are satisfied, yields an equation for the phase $\varphi$

$$
\varphi,{ }_{z}(z, t)+[\varphi, t(z, t)]^{2}=0 .
$$

Within its range of uniqueness the initial value problem in $z$ for (28) may readily be solved by the method of characteristics. Let $t_{0}$ be a solution of

$$
t=t_{0}+2 \varphi, t\left(0, t_{0}\right) z .
$$

Then, as long as the solution of (29) is unique, (28) is satisfied by

$$
\varphi(z, t)=\varphi\left(0, t_{0}\right)+\left(t-t_{0}\right)^{2} /(4 z) .
$$

The apparent singularity of (30) at $z=0$ is removable for initial conditions in $\mathcal{C}^{2}$, namely it follows from (29) that $t-t_{0}=O(z)$ when $z \rightarrow 0$ along a characteristic.

In a similar way to the third harmonic case [4], not only characteristics of the phase equation (28) are straight lines (29) and $\varphi_{t}$ is constant along these characteristics. Due to connection of $\varphi, t$ with squares of the amplitudes $G \times G^{*} / F^{2}$ and $H H^{*} / F^{2}$ by Eqs. (25), also the amplitudes do not vary along the same rectilinear characteristics. As a result the whole solution is constant along some straight lines. This is the typical property of single solitary waves rather than multisoliton solutions, in which interaction between solitons should result in a distortion of their paths. Hence we conclude that the only possible soliton solutions are single solitary waves.

When the conditions (23) and the first choice of (24) together with (18) are substituted to the initial equations (6), if the phase $\varphi$ satisfies (28), both equations (6) yield identical equations for the modulus $u=|U|$ and the phase $\varphi$

$$
\begin{aligned}
& u,{ }_{t t} \pm \sqrt{2} u^{2}=0, \\
& u,_{z}+2 u,_{t} \varphi,_{t}+u \varphi,{ }_{t t}=0,
\end{aligned}
$$

where the plus sign in front of $\sqrt{2}$ in (31) corresponds to $\psi=2 \varphi+2 k \pi$ in (23) while the minus sign appears when we choose $\psi=2 \varphi+(2 k+1) \pi$. Note that these two variants of sign in (31) have nothing to do with the previous sign doubling in (6) and (20). Both versions of (31) follow from one system of equations, namely (6) with $P=1 / 2$ and the plus sign in front of $U^{2}$. A sum of (32) multiplied by i with (31) cast these two real equations in the form of one complex equation, which might be obtained directly from the first one of (6)

$$
\mathrm{i} U,_{z}+U_{, t t} \pm \sqrt{2}|U| U=0 .
$$

A similar equation for $W$ which follows from the second of (6) is equivalent to a 
sum of (32) multiplied by i/2 and (31). This equation belongs to the nonlinear Schrödinger (NLS) type. To our best knowledge it has not been described in the literature so far (except for general works on the whole NLS family).

\section{The travelling wave solution}

The results of the previous section constitute a basis for a search for single travelling wave solutions of (6). The travelling wave solution may be obtained directly by assuming that $u$ depends merely on a combined variable $\tau=t-z / v$ (where $v$ is interpreted as the velocity of the wave), while the phase $\varphi$ may depend on both $z$ and $\tau$ independently, except for the constraint following from (28). At all points where $u \neq 0$ the solution of $(32)$ is

$$
\varphi=\tau /(2 v)+z /\left(4 v^{2}\right)+\varphi_{0}=t /(2 v)-z /\left(4 v^{2}\right)+\varphi_{0},
$$

where $\varphi_{0}$ is a constant.

The amplitude $u$ is a solution of

$$
u,_{\tau \tau} \pm \sqrt{2} u^{2}=0 .
$$

This system may readily be integrated once, yielding

$$
u_{\tau}^{2}= \pm(2 \sqrt{2} / 3)\left(u_{0}^{3}-u^{3}\right),
$$

where $u_{0}$ is a constant of integration.

One should bear in mind the meaning of $u$ as a modulus of a complex function. Due to this interpretation only nonnegative values of $u$ have a physical sense. At the points where $u=0$ the derivative $u_{\tau}$ changes sign while the phase $\varphi$ jumps by $\pi$ (note that the continuous solution (34) is limited to such values of $\tau$ for which $u \neq 0)$. Eventually the solution is proportional to the Weierstrass $\wp$ function

$$
u(\tau)=\mp 3 \sqrt{2} \wp\left(\tau-\tau_{0} ; 0, \mp \sqrt{2} u_{0}^{3} / 27\right),
$$

in the interval of positive $u$, within one real period of $\wp$; then it is periodically repeated to other values of $\tau$ ( $\tau_{0}$ is the second constant of integration).

These solutions to (36) are physically sensible, i.e. finite, only for the version of (35-37) with the upper sign. Their period may increase indefinitely on increasing of $u_{0}$; it tends to 0 when $u_{0} \rightarrow 0$. Thus the solution has the form of a periodic wave train without a proper soliton limit.

\section{Conclusion}

We have examined the second harmonic equations using the Painlevé test and the Hirota bilinear formalism. The Painlevé test has shown that in general the system does not have the Painlevé property, furthermore it has pointed out special values of the ratio of dispersion coefficients for which the system is partially integrable in the sense of Painlevé. The Hirota bilinear formalism limits these possibilities even further: the two conditions which it yields, one for the phases and another one for the amplitudes of the fundamental mode and its second harmonic, 
allow for nontrivial solutions iff their ratio of dispersion coefficients $P:=D_{1} / D_{2}$ $=2$. For this value of $P$ we have obtained the explicit solution for the phase by the method of characteristics. These characteristics, which also prove to be the lines of constant amplitude, are straight lines. As such evolutions do not leave room for soliton interaction, we conclude that the only possible soliton solutions are single envelope waves of the nonlinear Schrödinger type with the phase increasing linearly both in $z$ and $t$. We have found such solutions: they have the shape of a train of envelope periodic travelling waves rather than solitons. Their amplitude is proportional to the absolute value of the Weierstrass $\wp$ function in an interval where this function is negative, their values are repeated periodically elsewhere. They rather seem to be special solutions; more general soliton-like waves may exist which exceed the scope of the Hirota bilinear formalism. A thorough analysis of the system (3) by means of generalizations of the Hirota method [17] may help to go beyond these limitations.

\section{References}

[1] P.A. Franken, A.E. Hill, C.W. Peters, G. Weinreich, Phys. Rev. Lett. 7, 118 (1961).

[2] M.A. Karpierz, M. Sypek, Opt. Commun. 110, 75 (1994).

[3] A.V. Buryak, Yu.S. Kivshar, Phys. Lett. A 197, 407 (1995).

[4] V. Cao Long, P.P. Goldstein, M. Trippenbach, Acta Phys. Pol. A 105, 437 (2004).

[5] J. Weiss J. Math. Phys. 25, 2226 (1984).

[6] R. Conte, in: The Painlevé Property One Century Later, Ed. R. Conte, Springer Verlag, New York 1999, p. 77.

[7] C. Etrich, U. Peschel, F. Lederer, B.A. Malomed, Phys. Rev. E 55, 6155 (1997).

[8] R. Hirota, in: Solitons, Eds. R.K. Bullough, P.J. Caudrey, Springer-Verlag, Berlin 1980, p. 157.

[9] S.A. Akhmanov, A.P. Sukhorukov, R.V. Khokhlov, Usp. Fiz. Nauk 93, 19 (1967); Sov. Phys.- Usp. 10, 609 (1968).

[10] S. Kovalevskaya Acta Math. 12, 177 (1889), 14, 81 (1890).

[11] J. Weiss, M. Tabor, G. Carnevale, J. Math. Phys. 24, 522 (1983).

[12] M. Musette, in: The Painlevé Property One Century Later, Ed. R. Conte, Springer Verlag, New York 1999, p. 517.

[13] P.P. Goldstein, in: Proc. First Non-Orthodox School on Nonlinearity and Geometry, Warsaw, September 1995, Warsaw, Eds. D. Wójcik, J. Cieśliński, Polish Scientific Publishers, Warsaw 1998, p. 207.

[14] M. Jimbo, M.D. Kruskal, T. Miwa, Phys. Lett. A 92, 59 (1982).

[15] M.D. Kruskal, in: Painlevé Transcendents, Proc. NATO Advanced Research Workshop, September 1990 Sainte-Adele (Quebec) Canada, Eds. D. Levi, P. Winternitz, Plenum, New York 1992, p. 187.

[16] R. Conte, A.P. Fordy, A. Pickering, Physica D 69, 33 (1993).

[17] J. Hietarinta, Int. J. Mod. Phys. A 12, 43 (1997); B. Grammaticos, A. Ramani, J. Hietarinta, Phys. Lett. A 190, 65 (1994). 